\title{
Costing health care procedures: Art or science?
}

\author{
Gillian R Currie PhD ${ }^{1,3}$, Braden Manns MD MSc FRCPC 2,3
}

G iven that costing studies are not commonly published $\mathbf{J}_{\text {in clinical journals, it is important to preface specific }}$ comments about the article by Crott et al appearing in the present issue of The Canadian Journal of Gastroenterology (pages 473-482) with a general comment about the role of costing studies and, more broadly, of economic evaluation in health care. In aiding the resource allocation decisions in the planning of health services, the aim of economic evaluation is to ensure that the benefits from health care programs implemented are greater than the 'opportunity costs' of such programs. The concept of opportunity cost, which is central to health economics, rests on two principles scarcity of resources and choice (1). With regard to scarcity, even in societies with great wealth, there are not enough resources to meet all desires, particularly in the face of expensive technological advancement. Making choices in the context of resource scarcity means that decisions must be made regarding which health programs to fund and, therefore, which ones to forgo. It is the benefits associated with forgone health care programs that constitute opportunity costs. To make choices in health care that minimize the opportunity costs, information about both the costs and the benefits associated with alternate uses of those resources is required. Thus, a high quality costing study is one part of an economic evaluation. To some extent, the decision regarding what to include in such costing studies is a matter of expert judgment of the analysts, and thus an art. However, such judgments rely on some fundamental principles, such as opportunity cost. Therefore, it is science as well as art, which means that there is scope for debate about what costs should be 'in' and what costs should be 'out'.

Most clinicians are used to interpreting the measurement of health benefits (ie, improvements in survival or quality of life) but are unfamiliar with the measurement of costs. The study by Crott et al aimed to obtain an accurate estimate of the cost of an upper gastrointestinal endoscopy. Different methods can be employed to determine the cost of health care procedures $(2,3)$. Many studies have equated the price charged for a procedure (particularly in an American context) with the cost of a procedure (the actual cost of labour and supplies necessary for a procedure). This method has been criticized because it does not take into

\footnotetext{
${ }^{1}$ Department of Economics, ${ }^{2}$ Department of Medicine, and ${ }^{3}$ Department of Community Health Sciences, University of Calgary, Calgary, Alberta Correspondence: Dr Gillian R Currie, 2500 University Drive NW, SS 542, Calgary, Alberta T2N 1N4. Telephone 403-220-5602, fax 403-282-5262, e-mail currie@ucalgary.ca
} 
account the true 'opportunity cost' of using those resources. Alternatively, some studies have used macrocosting approaches, whereby the budget for an entire health program (ie, gastrointestinal scoping unit) is allocated to the different procedures that are performed within the unit (ie, a top down approach). This method is associated with problems such as how to allocate shared resources to different procedures accurately.

Crott et al used an activity-based costing methodology to determine the cost of an upper endoscopy. By using this method, every activity that is required to perform an upper endoscopy is determined, and each activity is then costed using a microcosting approach. Using this costing approach, the authors determined that, in their setting (Montreal, Quebec), the cost of an upper endoscopy ranges from $\$ 62$ for an unsedated and unbiopsied patient to $\$ 89$ for a sedated and biopsied patient (excluding the physician fees and reimbursement fee for biopsy analysis) (3). Including hospital overhead costs would add an additional $\$ 41$ per endoscopy; using nonreusable biopsy forceps for a biopsied patient would add a further $\$ 63$.

This is one of the first costing studies that has focused on the cost of an upper endoscopy. This type of costing analysis is complex, and the authors have done a good job of estimating the cost of the supplies and equipment needed to perform an upper endoscopy. As noted above, there is some judgment involved in determining what to include in a costing study. While the authors indicate that the study is part of a larger economic evaluation project, they do not provide details on the specific study question. If the purpose of the broader economic evaluation had been provided, it would have been easier to evaluate the choices made in this case. Some potential issues that affect the generalizability of this study include the costing of nursing time, including nursing administration; assumptions regarding the reuse of biopsy forceps; the opportunity cost of the building; and the allocation of overhead costs.

For instance, the authors only costed the time that the endoscopy nurse spent directly with the patient. It is likely that the total wages of the nurse were not fully accounted for by considering only the time spent directly with patients. It would have been more useful if the authors had taken into account the total wages of the endoscopy unit and allocated these wages based on the volume and complexity of services provided within the unit. Moreover, sedated patients may require monitoring for longer periods than suggested in this study, which would increase the cost of a sedated upper endoscopy. Some kind of sensitivity analysis (see below), attempting to account for such local differences in key variables, would have helped to make the study results more generalizable.

Crott et al raise the issue of the high cost of the biopsy forceps (almost equivalent to the cost of the endoscopy itself). It is important to highlight that the findings of a cost study alone are not sufficient for making informed choices about how to deliver care in a clinical area; data on both costs and outcomes are required to provide useful informa- tion. Specifically, the cost savings that would be associated with reusing instruments must be tempered against the potential health concerns associated with this reuse (4).

Given the age of the building in which the endoscopy suite is housed, the authors assumed full depreciation and, therefore, excluded any building costs in their analysis. This approach failed to take into account the notion of opportunity cost, whereby in the absence of an endoscopy suite, the space could presumably be used by another health-enhancing activity (ie, a rehabilitation program for patients with lung disease).

The authors also raise the issue of allocation methods for overhead costs. How to allocate these costs depends on the nature of the context within which the study is being conducted. If a hospital has spare capacity, the marginal cost of an extra procedure may not need to reflect any extra overhead costs. However, if no spare capacity exists, then building costs and additional overhead costs would need to be considered (1).

The points listed above all relate to the external generalizability of these results. For instance, nursing costs may differ significantly across the country. Moreover, this hospital appears to perform a higher proportion of unsedated upper endoscopies than might be considered the norm in Canada. Given the cost savings, hospitals may want to investigate the use of (and clinical outcomes associated with) unsedated endoscopies; however, this approach may limit the generalizability of these results. With respect to the building costs, particularly for hospitals that are considering building or expanding an endoscopy suite, the opportunity cost of this space must be valued in some sense.

One method of addressing these concerns would be to perform a complete sensitivity analysis. In a sensitivity analysis, uncertain variables (ie, such as the time required for the nurse or the recovery of the patient) are varied within clinically plausible ranges to determine their effect on the estimates of costs. This would also allow the authors to investigate the sensitivity of their results to assumptions regarding the depreciation rate and the lifespan of equipment. The authors point out that earlier costing studies were quite sensitive to these two variables; thus, their study could have addressed these in a careful sensitivity analysis. A careful sensitivity analysis can provide information about the internal validity of the results, and would enable others to determine how the results of this study would apply within their specific context.

Broadly speaking, high quality costing studies are important because they are needed for economic evaluations in the area of gastrointestinal medicine. Economic evaluations are studies that compare alternative strategies for the treatment and diagnosis of a medical condition on the basis of their impact on both costs and health benefits. Economic evaluations can help answer two types of (related) questions (1). The first question is how best to deliver a particular health program (ie, "With a fixed amount of resource available, what is the most efficient way of investigating patients who present with dyspepsia, noninvasive testing 
for Helicobacter pylori or endoscopy plus $H$ pylori testing" (5). The second type of question concerns how to determine which health-enhancing programs should receive health resources (ie, an endoscopy unit wishing to expand its services competes for funding with a respiratory program wishing to establish a rehabilitation program for patients with chronic obstructive pulmonary disease). Economic evaluation can provide the types of information needed to address both of these important questions.

Given the scarcity of health care resources and that new and effective therapies are continually being developed for patients with gastrointestinal disease, costing studies and economic evaluations will play an increasingly important role in determining the appropriate use of new therapies.

\section{REFERENCES}

1. Donaldson C, Shackley P. Economic evaluation. In: Detels R, Holland WW, McEwan J, Omenn GS, eds. Oxford Textbook of Public Health, 3rd edn. Oxford: Oxford University Press, 1997:849-70.

2. Edbrooke D, Stevens V, Hibbert C, Mann A, Wilson A. A new method of accurately identifying costs of individual patients in intensive care: the initial results. Int Care Med 1997;23:645-50.

3. Noseworthy TW, Konopad E, Shustack A, Johnston R, Grace M. Cost accounting of adult intensive care: Methods and human and capital inputs. Crit Care Med 1996;24:1168-72.

4. Muscarella LF. Biopsy forceps: disposable or reusable? Gastroenterol Nurs 2001;24:64-8.

5. McColl KEL, Murray LS, Gillen D, et al. Randomised trial of endoscopy with testing for Helicobacter pylori compared with non-invasive $H$ pylori testing alone in the management of dyspepsia. BMJ 2002;324:999-1002. 


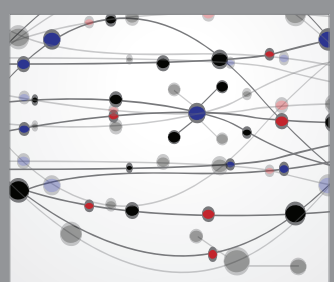

The Scientific World Journal
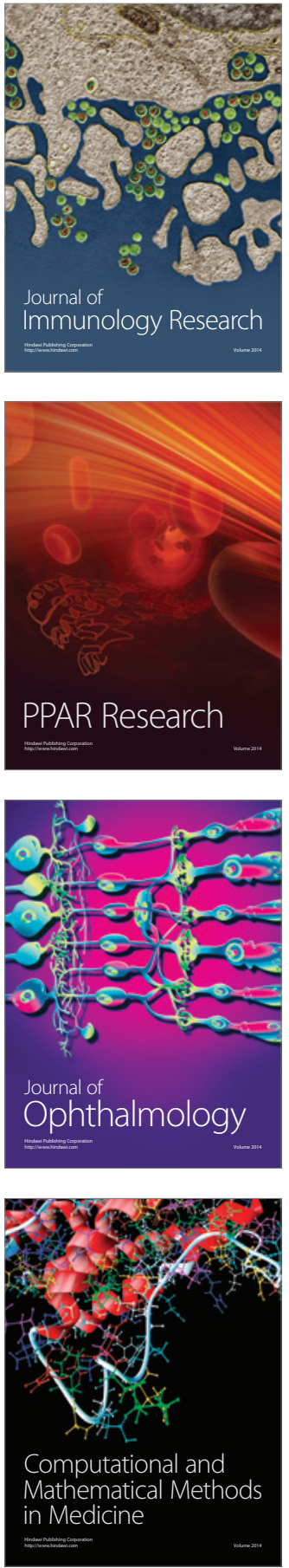

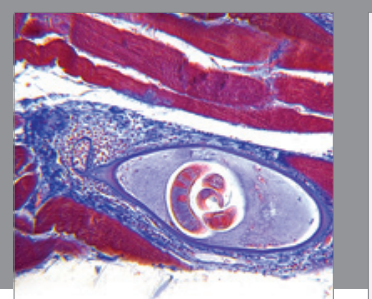

Gastroenterology Research and Practice

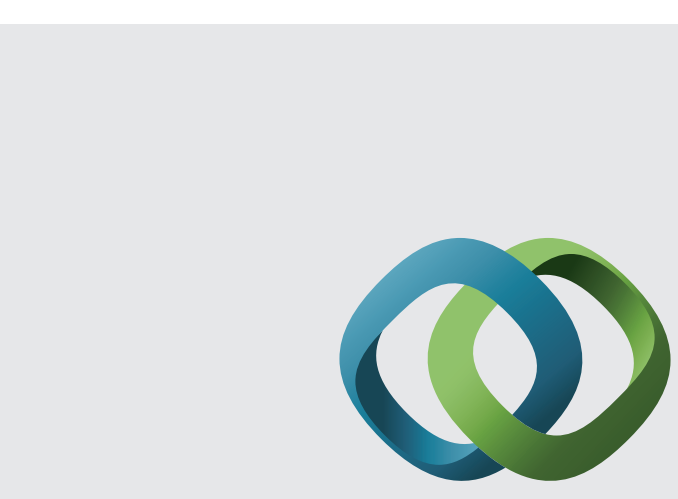

\section{Hindawi}

Submit your manuscripts at

http://www.hindawi.com
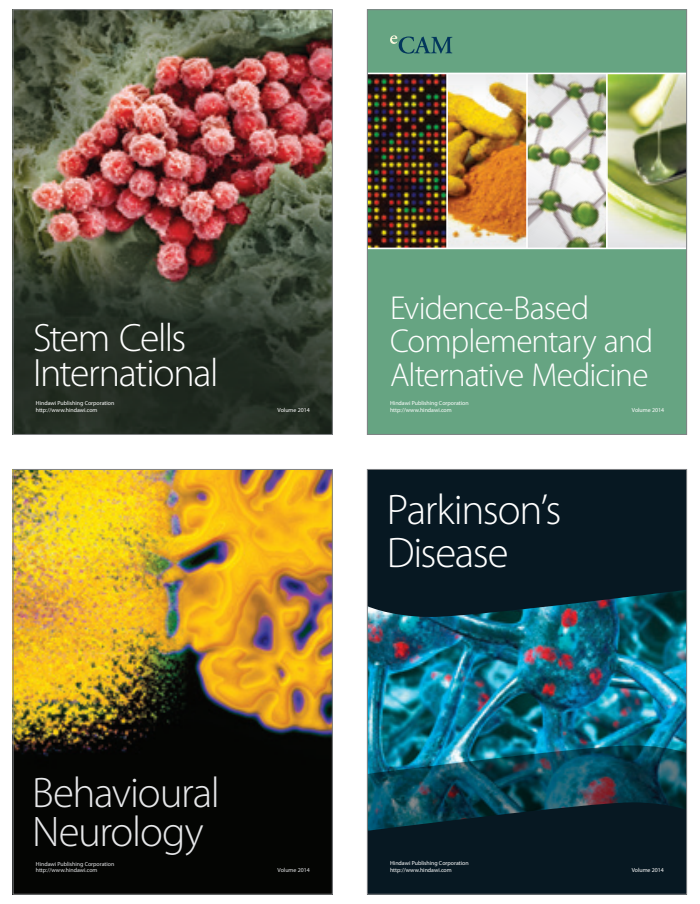
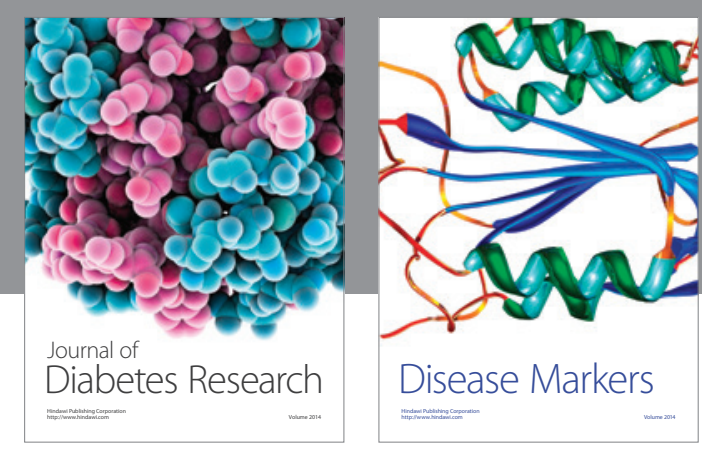

Disease Markers
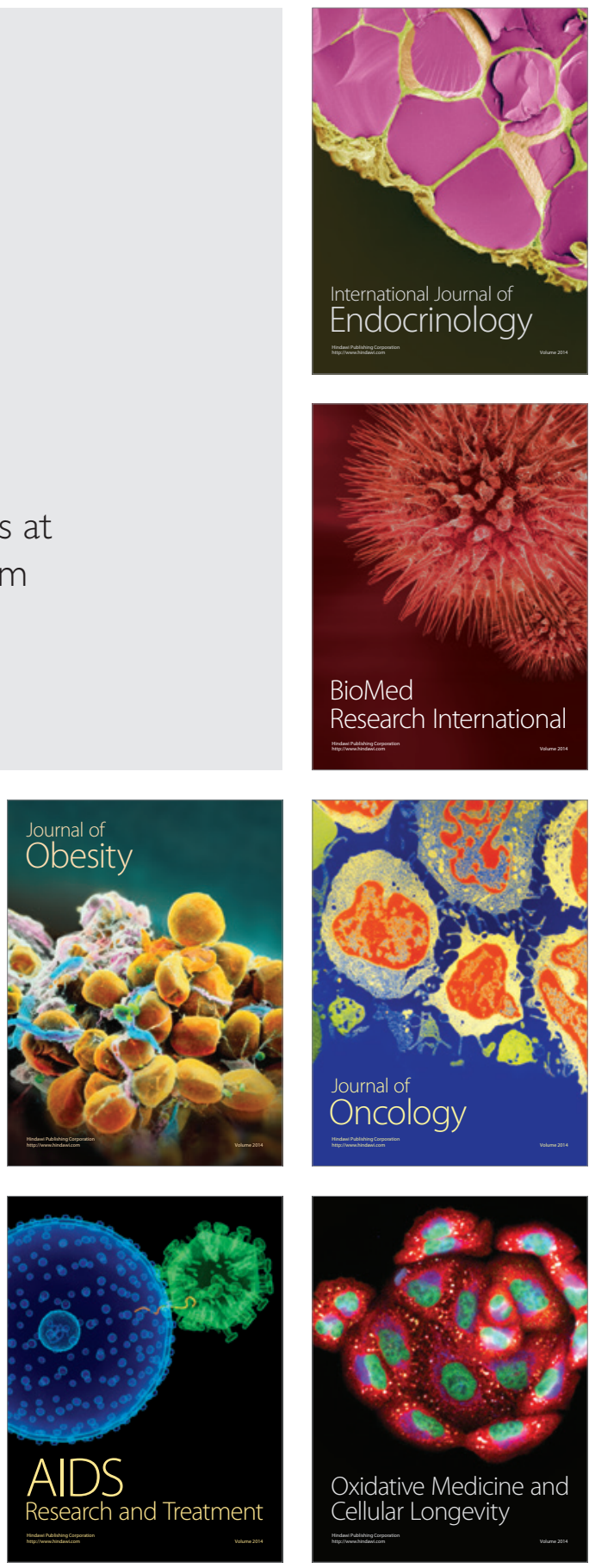